\title{
La microstéréolithographie et ses applications
}

\author{
Serge Monnereta \\ Institut Fresnel, Domaine universitaire de Saint-Jérôme, 13397 Marseille Cedex 20, France
}

Reçu le 15 mai 2005

\begin{abstract}
Résumé - Cet article a pour but de donner un aperçu des différents procédés de microstéréolithographie développés au niveau international. En effet, ces techniques connaissent actuellement un développement notable, grâce à des avancées technologiques importantes effectuées dans le domaine des afficheurs UV à micromiroirs, mais aussi grâce au contrôle de l'absorption à deux photons. Les applications qui en découlent concernent le micro-prototypage rapide, la micromécanique, mais aussi la microfluidique et de manière plus générale la réalisation de microcomposants tridimensionnels de formes complexes, y compris en matériaux frittés.
\end{abstract}

Mots clés : Stéréolithographie / microtechnologies / prototypage rapide / photopolymère

\begin{abstract}
The purpose of this paper is to give an outline of the various processes of microstereolithography developed at the international level. Indeed, these techniques currently know a strong development, thanks to important technological projections carried out in the field of UV micromirrors-based displays, but also thanks to the control of two-photons absorption. The applications which result from this relate to rapid prototyping, micromechanics, but also microfluidics, and in a more general way to the realization of three-dimensional microcomponents of complex shape, including sintered microparts.
\end{abstract}

Key words: Stereolithography / microtechnologies / rapid prototyping / photopolymer

\section{Introduction}

Le terme de stéréolithographie décrit un procédé qui permet en quelques heures d'obtenir sans aucun usinage, un modèle de pièce mécanique à partir des données contenues dans un système de CFA0. Le processus (Fig. 1) consiste en la CAO (ou/et la numérisation 3D d'un objet existant), la phase de calcul (tranchage numérique) puis la mise en forme effective par la machine de fabrication, à partir d'un procédé fonctionnant par ajout de matière, en couches successives. Le procédé classique, maintenant largement industrialisé, permet de fabriquer des objets volumiques de formes complexes, généralement pour des applications de prototypage rapide. Il permet en effet ces réalisations avec des temps de fabrication courts, et suivant un processus totalement informatisé et automatique.

Le processus de fabrication repose sur une réaction chimique de photopolymérisation : une résine monomère photosensible liquide se transforme localement en un polymère solide sous irradiation lumineuse. Si l'éclairement

\footnotetext{
a Auteur correspondant : serge.monneret@fresnel.fr
}

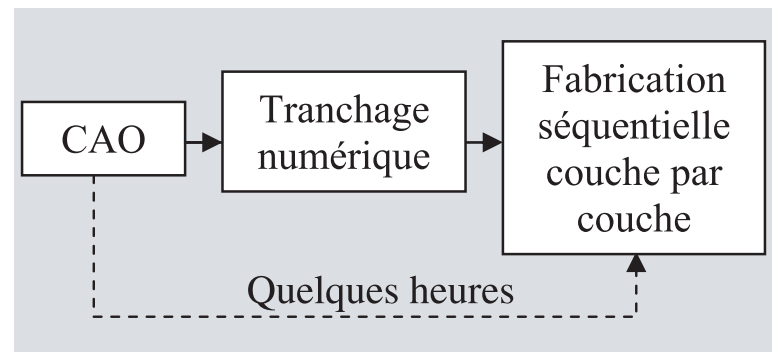

Fig. 1. Les étapes principales de la stéréolithographie.

est tel qu'il induit un motif géométrique sur la surface de résine éclairée (par balayage ou par projection d'image), alors la couche de polymère formée reproduira ce motif. La répétition séquentielle d'étapes de mises en couche de résine suivies d'insolations structurées de ces dernières va conduire à former des objets tridimensionnels de formes complexes, par empilements successifs des différentes tranches de l'objet les unes sur les autres. 
Chaque photon absorbé conduit à la création d'un centre actif radicalaire qui réagit avec le liquide photosensible pour former une chaîne polymère, par une réaction en chaine. Cette réaction se termine lorsque le milieu est suffisamment polymérisé, donc visqueux, pour empêcher les réactifs de se rencontrer. La longueur typique des chaînes polymères formées est alors nettement en dessous du micromètre, ce qui fait l'intérêt majeur d'une initiation photonique : si le milieu ne diffuse pas la lumière, alors le volume solidifié suit directement la distribution lumineuse spatiale du faisceau excitateur. Cette propriété est à la base du contrôle dimensionnel des objets fabriqués.

En conséquence, il est possible de mettre en forme des objets avec une résolution micrométrique en optimisant les caractéristiques de l'éclairement lumineux. Plusieurs grandes approches ont alors été suivies pour tenter de fabriquer des micro- ou même nano-composants par photopolymérisation, qui se distinguent essentiellement par les caractéristiques du système d'éclairement utilisé, ainsi que par les processus physiques de photo-polymérisation mis en jeu. Dans tous les cas, le terme générique de microstéréolithographie (microSL) est communément retenu pour englober ces procédés.

Le but de cet article est tout d'abord de présenter les travaux effectués dans le domaine de la microSL au niveau international, puis de donner un aperçu de leurs applications réelles ou potentielles. La description des procédés est divisée en deux parties, suivant que la réaction de photopolymérisation est générée par un processus d'absorption à un ou à deux photons. En effet, le processus de très loin le plus probable d'interaction d'un photon rencontrant un matériau présentant une bande d'absorption correspondant à son énergie, est l'absorption à un photon. C'est en conséquence le processus qui initie les réactions de photopolymérisation de la quasitotalité des procédés de micro-stéréolithographie actuels. Cependant, l'absorption à deux photons ouvre des perspectives très intéressantes en matière de résolution des objets fabriqués, d'où l'apparition très récente de quelques procédés basés sur ce type d'absorption.

\section{Les procédés « monophotoniques »}

On distingue deux grandes familles dans les procédés de microSL fonctionnant sur ce type de photopolymérisation. La première famille est basée sur une écriture dite « vectorielle » des objets, c'est-à-dire par balayage d'un faisceau laser focalisé sur la surface du matériau photopolymérisable. Nous verrons que les microobjets fabriqués par ces procédés peuvent présenter une excellente résolution, sous condition d'être limités à des tailles nettement inférieures au millimètre. Cependant, la plupart des procédés actuels fonctionne plutôt avec des masques, qui permettent la fabrication globale d'une couche en une seule illumination, les objets étant toujours constitués d'un empilement de couches. Les procédés de cette deuxième famille présentent l'avantage non négligeable d'être plus rapides que les procédés à écriture vectorielle des couches, puisque l'irradiation

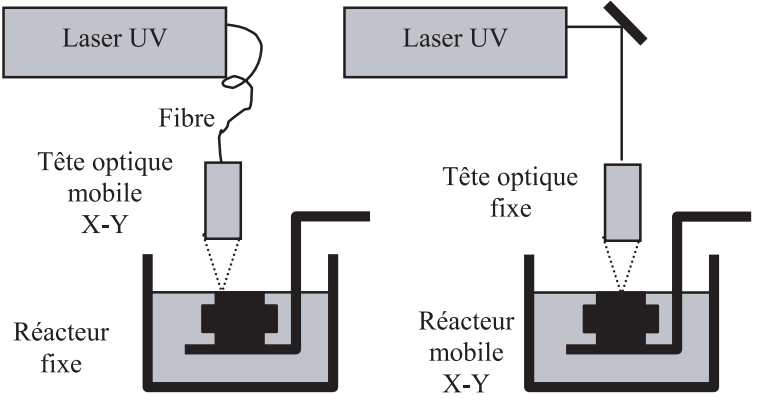

Fig. 2. Les 2 principes retenus en microSL dans le cas des systèmes d'illumination vectorielle.

d'une couche est de durée courte et indépendante du motif à polymériser. De plus, la taille des objets fabriqués peut être de l'ordre de quelques millimètres, avec une résolution latérale pouvant dans certains cas atteindre $5 \mu \mathrm{m}$. Par contre, la résolution longitudinale obtenue avec ces procédés est nettement moins bonne que celle démontrée avec les procédés à écriture vectorielle.

\section{Les dispositifs vectoriels}

L'utilisation de miroirs galvanométriques pour la fabrication de pièces de volumes de quelques $\mathrm{mm}^{3}$ conduit à une résolution relativement faible provenant de la difficulté à focaliser un faisceau mobile en rotation sur une surface plane, tout en maintenant un diamètre de focalisation constant sur cette dernière. Pour dépasser ce problème, les premiers procédés de microSL proposés reposent sur la focalisation statique du faisceau lumineux, associée à un déplacement de la cuve contenant la résine photopolymérisable. Une autre solution, suivie par la société Meiko (Japon), consiste à déplacer la tête optique grâce à l'utilisation d'une fibre optique (Fig. 2).

Les deux procédés de microSL par écriture directe vectorielle d'un faisceau focalisé sur la surface d'une résine photopolymérisable les plus aboutis ont été mis au point au Japon. Le premier, développé au « Kyushu Institute of Technology » par Ikuta et al., consistait à irradier la résine à travers une vitre, à l'aide d'une lampe UV à Xénon dont le faisceau était focalisé sur une zone de diamètre $5 \mu \mathrm{m}$. Le second, développé à l'université de Nagoya par Nakamoto et al., comprenait une source laser de type He-Cd de longueur d'onde $325 \mathrm{~nm}$, de puissance typique $0,1 \mu \mathrm{W}$. Dans les deux cas, la résolution annoncée était de $5 \mu \mathrm{m}$ pour des pièces tri-dimensionnelles relativement complexes, mais limitées à des démonstrateurs. En effet, en raison des contraintes liées au déplacement de la cuve de liquide, la vitesse de balayage de cuve, typiquement égale à $30 \mu \mathrm{m} \mathrm{s}^{-1}$, rendait la durée de fabrication d'objets millimétrique économiquement rédhibitoire.

\section{Les dispositifs à masques}

L'illumination par masques permet au contraire de diminuer nettement les temps de fabrication, puisque l'irradiation d'une couche complète se fait par la projection d'une image bidimensionnelle sur la surface de la résine 


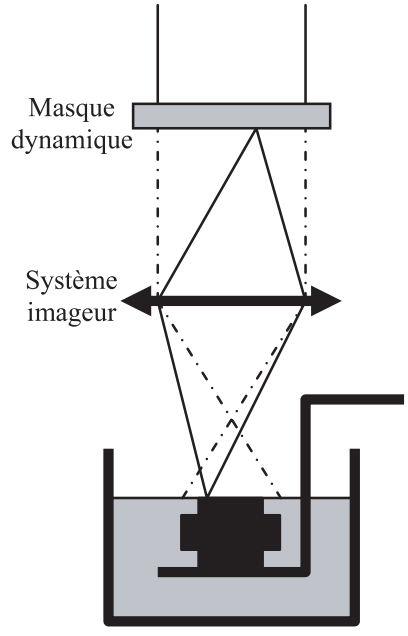

Fig. 3. MicroSL par masque dynamique.

photopolymérisable, de durée courte et indépendante du motif à polymériser. Ainsi, le micro-objet est toujours fabriqué couche par couche, mais avec un durcissement global et non plus vectoriel de chacune des couches.

Par contre, la résolution longitudinale est nettement moins bonne que celle proposée par les systèmes précédents, puisque seule l'absorption du matériau permet son contrôle : la profondeur de champ au niveau des images projetées est généralement nettement supérieure aux épaisseurs de couches.

La fabrication de pièces volumiques complexes par masquage nécessite un très grand nombre de masques différents, dès lors que ces pièces sont réellement volumiques et complexes. C'est pourquoi on utilise des masques dynamiques reconfigurables à volonté de manière électronique, plutôt que fabriquer des jeux de centaines de masques physiques qui rendraient le prix de revient des objets prohibitif.

Il ne reste donc dans le montage qu'un seul élément mobile, qui est la plate-forme élévatrice sur laquelle l'objet est fixé durant sa construction. La résolution latérale du procédé est alors beaucoup plus simple à contrôler, puisque les images sont parfaitement stabilisées d'une couche à l'autre (Fig. 3).

Le premier montage de microSL à masque dynamique a été mis au point à Nancy en 1996 par Bertsch et al. à partir d'un laser à argon ionisé émettant à 514,5 nm, combiné à un dispositif dynamique générateur de masques à cristaux liquides. L'intérêt de la microSL par projection directe d'images bidimensionnelles était démontré.

Les masques à cristaux liquides utilisés, seuls dispositifs alors disponibles pour permettre la projection dynamique de motifs sur la résine, présentent tout de même des inconvénients majeurs pour la microSL : ils sont opaques à l'ultra-violet (UV), ce qui nécessite le développement de résines spécifiques sensibles dans le domaine du visible, et présentent des caractéristiques optiques relativement mauvaises au niveau de la transparence et du contraste. En conséquence, de nombreux efforts ont dû être faits pour optimiser les procédés, en combinant au mieux les propriétés spectrales des sources lumineuses avec la sensibilité des photomatériaux d'une part et la transparence des masques d'autre part. Les résultats obtenus, par Bertsch et al. à Lausanne ou Monneret et al. à Nancy, ont permis de réaliser des pièces tridimensionnelles complexes en polymère avec une résolution de $5 \mu \mathrm{m}$ pour le montage suisse, ou céramique avec une résolution de $20 \mu \mathrm{m}$ pour les travaux français (voir paragraphe sur les applications).

Quelques travaux ont néanmoins eu pour objectif de développer des montages de microSL UV par masque dynamique à cristaux liquides. Ainsi, Farsari et al., en Grande-Bretagne, ont utilisé un masque optimisé pour l'UV, de résolution $800 \times 600$ pixels, associé à une source laser (Argon ionisé, longueur d'onde 351,1 nm). Quelques micro-objets tridimensionnels complexes de taille submillimétrique ont été réalisés grâce à ce procédé, avec des épaisseurs de couches allant de 40 à $5 \mu \mathrm{m}$. Cependant, en raison de la faible transmission des masques dans l'ultraviolet, les durées d'exposition peuvent atteindre la minute. Monneret et al. ont proposé un montage basé sur le phénomène physique de conversion de fréquence optique dans un cristal non linéaire, mais la résolution obtenue était moins bonne que celle de leur montage en lumière visible.

La grande amélioration des dispositifs de microSL par masque dynamique provient de l'avancée technologique opérée par Texas Instruments, qui a développé un afficheur à micro-miroirs (DMD®, système DLP®) de haute résolution et de haut contraste, compatible avec l'UV. Bertsch et al. à Lausanne ont été les premiers à insérer ce composant dans leur procédé, avec des résultats remarquables au niveau de la définition des objets fabriqués. Le montage actuel permet la fabrication d'objets en polymère pouvant atteindre une résolution transversale optimale de $3 \mu \mathrm{m}$. Des couches d'épaisseur $5 \mu \mathrm{m}$ peuvent être fabriquées en choisissant une densité d'énergie adaptée.

L'Institut Fresnel à Marseille, mais aussi le « Rutherford Appleton Laboratory » en Grande-Bretagne, développent actuellement des montages similaires avec une résolution de $1024 \times 768$ pixels.

\section{Les procédés « biphotoniques »}

Pour passer à une résolution sub-micronique des objets fabriqués, Kawata et al. ont appliqué le processus physique d'absorption à deux photons à la photopolymérisation d'objets solides tridimensionnels.

L'absorption à deux photons est un phénomène d'optique non linéaire ayant lieu dans tout matériau, dès lors que les niveaux d'irradiation sont suffisamment élevés pour que l'énergie combinée de deux photons permette la transition entre l'état fondamental et un état excité des molécules photosensibles. La dépendance quadratique de l'absorption à deux photons avec l'intensité lumineuse incidente permet de confiner cette transition à une zone fortement localisée sur le point de focalisation, et ainsi d'améliorer la résolution du procédé. 


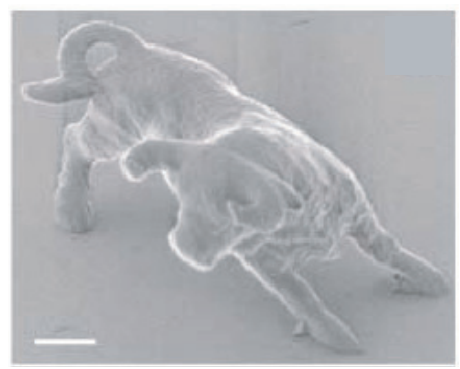

Fig. 4. Objet (10 $\mu \mathrm{m}$ de long, $7 \mu \mathrm{m}$ de haut) réalisé par microSL à deux photons (Kawata et al., université d'Osaka, Japon).

Pour se placer en régime d'absorption à deux photons, la puissance instantanée du faisceau laser doit être de plusieurs kilowatts. La source utilisée est alors un laser Tisaphir (longueur d'onde : $790 \mathrm{~nm}$; largeur d'impulsion : $150 \mathrm{fs})$ pulsé à une fréquence de répétition de $76 \mathrm{MHz}$, délivrant une puissance moyenne typique de $30 \mathrm{~mW}$. Le faisceau est focalisé, à l'aide d'une lentille, à l'intérieur d'une résine photopolymérisable dans l'UV. Le balayage bidimensionnel du faisceau laser est assuré par deux miroirs mobiles perpendiculaires. La cuve est supportée par un plateau mobile permettant l'obtention de la troisième dimension.

Cette méthode de micro-fabrication a permis de réaliser des structures en trois dimensions de taille très réduite (Fig. 4).

La résolution spatiale du procédé est de $120 \mathrm{~nm}$. Cependant, la taille maximale démontrée des objets est de $75 \mu \mathrm{m}$ dans la direction de l'axe optique. Cette limitation vient essentiellement des aberrations sphériques provoquées lors de la traversée des différents milieux air/cuve/résine. Là encore, l'utilisation d'un balayage du faisceau rend la durée de fabrication longue (3 heures pour l'objet de la Fig. 4). C'est pourquoi Kato et al. ont très récemment proposé un procédé à base de microlentilles permettant la fabrication parallèle de plus de 200 répliques, par multiplication des faisceaux laser focalisés dans la résine.

\section{Applications de la microSL}

La grande force du procédé de microSL est de permettre la fabrication d'objets tridimensionnels de formes complexes. Cependant, l'intérêt de la technique repose aussi sur les avancées faites au niveau des matériaux mis en forme : depuis quelques années, sous l'impulsion de l'équipe du Département de Chimie Physique des Réactions de Nancy, la microSL permet de réaliser des pièces en matériaux frittés (alumine, PZT, cuivre), ouvrant de nouvelles perspectives.

\section{Matériaux polymères}

Une grande application de la microSL de polymères est le prototypage rapide de microcomposants de formes

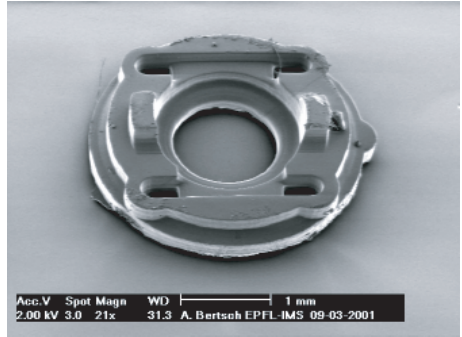

Fig. 5. Pièce prototype réalisée par microSL à masque à micro-miroirs (Bertsch et al., EPFL, Lausanne, Suisse).

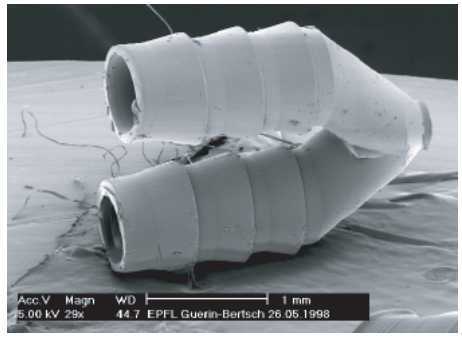

Fig. 6. Connecteur pour fluidique réalisé par microSL à masque à micro-miroirs (Bertsch et al., EPFL, Lausanne, Suisse).

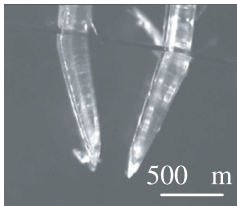

(a)

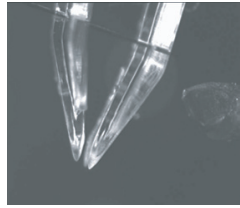

(b)

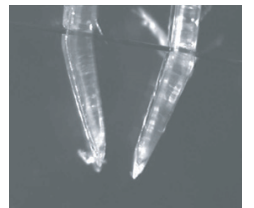

(c)
Fig. 7. Cycle de fermeture/ouverture d'une micro-pince en polymère, réalisée par microSL à masque à cristaux liquides (Loubère et al., DCPR, Nancy).

complexes (exemple Fig. 5). Par ailleurs, le design de petites pièces précises intéresse fortement les industries de la bijouterie et de l'horlogerie, qui demandent des états de surface compatibles avec les épaisseurs de couches offertes par la microSL (typiquement $10 \mu \mathrm{m}$ ).

Les matériaux polymères sont aussi de bons candidats pour la microfluidique. Non conducteurs, ils permettent de générer des flux électrophorétiques, et donc le pilotage de biomolécules ou autres entités chargées dans des canaux. De plus, la microSL permet d'intégrer à ces canaux les zones de connexion avec le monde macroscopique, ou de fabriquer directement des connecteurs externes (Fig. 6).

Enfin, il est possible d'insérer des éléments (fibres optiques, fils en alliage à mémoire de forme) en cours de fabrication afin d'intégrer des fonctions spécifiques à l'objet fabriqué. Ainsi, on peut réaliser des actionneurs pilotés par des fils en alliage à mémoire de forme (exemple d'une pince sur la Fig. 7), ou directement réaliser des structures sur des composants électroniques (Mizukami et al., Tokyo, Japon).

Bien sûr, d'autres applications proviendront du développement actuel de photopolymères aux propriétés 


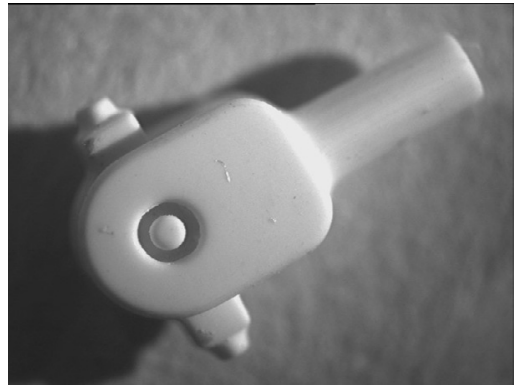

Fig. 8. Pièce mobile en polymère chargé en alumine, non frittée (longueur $3,5 \mathrm{~mm}$ ), réalisée par microSL à masque à cristaux liquides (Monneret et al. 2004, DCPR, Nancy).

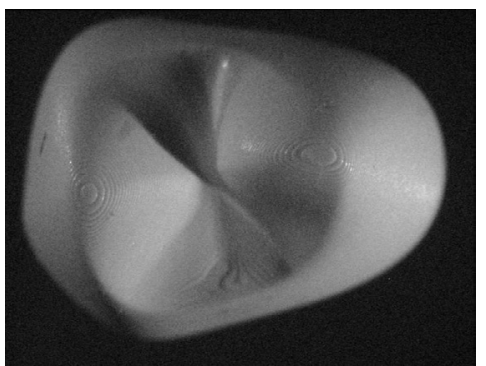

Fig. 9. Modèle de dent humaine en polymère chargé en alumine, ici non frittée (longueur 11,5 mm), réalisée par microSL à masque à cristaux liquides (réalisé par Monneret, DCPR, Nancy).

spécifiques (transparence, souplesse, couleur, ...), compatibles avec la mise en forme par microSL.

\section{Matériaux chargés - matériaux frittés}

La réalisation non plus de modèles de pièces mais de composants totalement fonctionnels entre dans le domaine du possible grâce au développement effectué sur les matériaux chargés mis en forme par microSL puis frittés. Trois étapes principales sont nécessaires pour obtenir une pièce frittée :

- la préparation de la suspension (résine + matériau en poudre + amorceur),

- la mise en forme par stéréolithographie de la pièce composite (polymère chargé en poudre de matériau),

- l'étape thermique conduisant à une pièce en matériau fritté.

La mise en forme de pièces chargées non frittées peut déjà s'avérer intéressante pour les applications en micromécanique ou microrobotique, en raison de la solidité et de la résistance accrues de composants par exemple chargés en poudre céramique (Fig. 8).

La fabrication de pièces céramiques (alumine, PZT, céramique bio-compatible, sont les matériaux développés au DCPR de Nancy) ouvre de nouvelles perspectives au niveau des applications, en particulier dans le domaine biomédical (prothèses, dents, voir Fig. 9).

Les pièces céramiques frittées présentent aussi l'intérêt de ne pas présenter de fluorescence parasite, contrairement à la plupart des polymères comprenant des

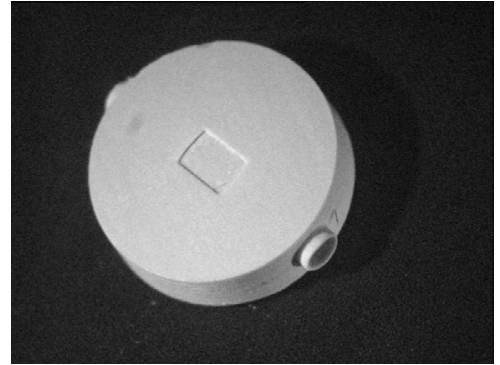

Fig. 10. Pièce en alumine frittée pour l'analyse de fluorescence en milieu liquide (diamètre $4 \mathrm{~mm}$ ), réalisée par microSL à masque à cristaux liquides (Provin et al. 2003, DCPR, Nancy).

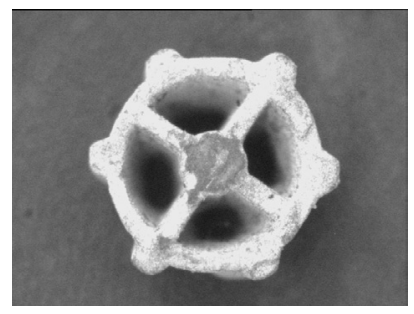

Fig. 11. Pièce en cuivre, frittée (diamètre $3,5 \mathrm{~mm}$ ), réalisée par microSL à masque à cristaux liquides (réalisé par Tasso, DCPR, Nancy).

photoinitiateurs fluorescents nécessaires à la photopolymérisation. Ainsi, la pièce de la figure 10 a été développée pour des applications d'analyse biologique par mesure de fluorescence sous flux électrophorétique (collaboration DCPR et Institut Fresnel, Marseille).

Par ailleurs, la réalisation de pièces en cuivre fritté (Fig. 11) ouvre d'autres perspectives, comme potentiellement la fabrication d'électrodes complexes pour l'usinage par électroérosion. Pour cela, les états de surface des objets fabriqués doivent encore être améliorés, afin de permettre la création de moules métalliques utilisables directement pour l'injection plastique de micro-composants.

Enfin, on peut associer la fabrication d'objets polymères par microSL à la réalisation de composants céramiques, par des procédés de transfert de type « moulage silicone », comme l'ont proposé Knitter et al. à Karlsruhe (Allemagne, Fig. 12). Dans ce cas, la pièce réalisée par microSL est le modèle initial qui permet de réaliser l'empreinte nécessaire à sa réplication en « bonne matière $»$.

\section{Conclusion}

L'évolution actuelle des procédés de microSL se concentre sur la fabrication par projection d'images bidimensionnelles au moyen de masques dynamiques à micromiroirs. Les pièces réalisables ont des volumes typiques variant du $\mathrm{mm}^{3}$ au $\mathrm{cm}^{3}$ avec une résolution pouvant atteindre $5 \mu \mathrm{m}$. La panoplie de matériaux compatibles avec ces procédés s'étend progressivement, pour permettre maintenant de travailler avec de nombreux types de polymères aux propriétés spécifiques, mais aussi avec des 


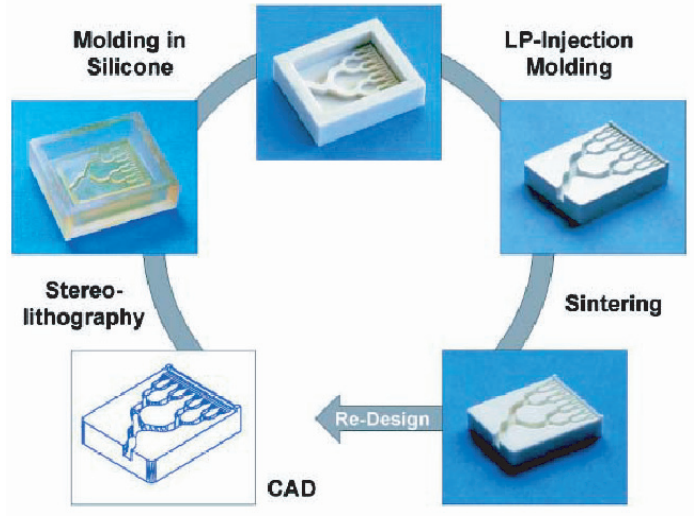

Fig. 12. Procédé de transfert polymère/céramique par moulage silicone (Knitter et al., Karlsruhe, Allemagne).

céramiques, et bientôt des métaux. Cette évolution doit conduire à une multiplication des machines de microSL, ce que l'on commence effectivement à vérifier, que ce soit dans les laboratoires de recherche comme dans l'industrie. Par ailleurs, l'apparition de montages biphotoniques permet d'envisager maintenant la « nanoSL », avec une résolution nettement sub-micronique.

\section{Références}

[1] A. Bertsch, P. Bernhard, P. Renaud, Microstereolithography: concepts and applications, Proceedings of 8th IEEE International Conference on Emerging Technologies and Factory Automation (ETFA 2001) 2 (2001) 289-298

[2] O. Dufaud, S. Corbel, Stereolithography of PZT ceramic suspensions, Rapid Prototyping Journal 8 (2002) 83-90

[3] M. Farsari, F. Claret-Tournier, S. Huang, C. Chatwin, D. Budgett, P. Birch, R. Young, J. Richardson, A novel highaccuracy micro-stereolithography method employing an adaptive electro-optic mask, J. Materials Proc. Technol. 107 (2000) 167-172
[4] K. Ikuta, T. Ogota, M. Tsubio, S. Kojima, Development of mass productive micro stereo lithography, Proc. of IEEE International Workshop on Micro Electro Mechanical Systems (MEM'96), 1996, pp. 301-306

[5] J. Kato, N. Takeyasu, Y. Adachi, H. Sun, S. Kawata, Multiple-spot parallel processing for laser micronanofabrication, Appl. Phys. Lett. 86 (2005) 044102

[6] S. Kawata, H.-B. Sun, T. Tanaka, K. Takada, Finer features for functional microdevices, Nature 412 (2001) 697-698

[7] R. Knitter, D. Göhring, P. Risthaus, J. Hausselt, Microfabrication of ceramic microreactors, Microsystems Technologies 7 (2001) 85-90

[8] V. Loubère, S. Monneret, H. Le Gall, S. Corbel, Microstereolithography using a dynamic mask for microactuators fabrication, Revue Internationale de CFAO et d'Informatique Graphique 15 (2000) 229-243

[9] Y. Mizukami, D. Rajniak, A. Rajniak, M. Nishimura, A novel microchip for capillary electrophoresis with acrylic microchannel fabricated on photosensor array, Sensors Actuators B 81 (2002) 202-209

[10] S. Monneret, H. Le Gall, V. Badé, F. Devaux, A. Mosset, E. Lantz, Dynamic UV microstereolithography, Eur. Phys. J. AP 20 (2002) 213-218

[11] S. Monneret, Microfabrication directe de pièces céramiques tridimensionnelles de formes complexes, Techniques de l'Ingénieur (2004)

[12] T. Nakamoto, K. Yamaguchi, P. Abraha, K. Mishima, Manufacturing of three-dimensional micro-parts by UV laser induced polymerization, J. Micromech. Microeng. 6 (1996) 240-253

[13] C. Provin, S. Monneret, H. Le Gall, H. Rigneault, P.F. Lenne, H. Giovannini, New process for manufacturing ceramic microfluidic devices for microreactor and bioanalytical applications, dans Microreaction Technology, Editeur : Springer Verlag, Berlin, 2001, pp. 103-112

[14] C. Provin, S. Monneret, H. Le Gall, S. Corbel, Threedimensional ceramic microcomponents made using microstereolithography, Advanced Materials 15 (2003) 994-997 